\title{
Gender-sensitive social protection A critical component of the COVID-19 response in low- and middle-income countries
}

\section{Melissa Hidrobo, Neha Kumar, Tia Palermo, Amber Peterman, and Shalini Roy}

\section{T} o grapple with the unfolding economic crisis triggered by COVID-19, governments are increasingly turning to social protection to mitigate against widespread economic downturn and to support vulnerable populations through times of health risk and economic scarcity. According to the World Bank, as of April 17, 133 countries ${ }^{1}$ had adapted or introduced approximately 564 social protection initiatives. ${ }^{2}$ Understandably, the primary concern of these social protection responses has been to provide rapid economic assistance; gender considerations have not been at the forefront. This is unsurprising, as most existing social protection programs in low- and middle-income countries (LMICs) are either gender-blind or gender-neutral at best. ${ }^{3}$

However, such programs may inadvertently exacerbate gender inequalities. ${ }^{4}$ In the context of the COVID-19 response, this is particularly worrying, given that the pandemic is likely to pose or intensify different risks for men and women, including potentially widening existing gender inequalities. ${ }^{5}$ Examples of risks include health risks (e.g., high risk of infection due to larger shares of women in the global health workforce, reproductive health risks, and maternal mortality due to overburdened health systems); loss of jobs and livelihoods (e.g., economic impacts are likely to be more severe for informal workers and women already earning comparatively less than men); increased, disproportionate burden of care among women; and protection risks for women and girls (e.g., increased school drop-outs, pregnancy, and early marriage among adolescent girls; increased risk of gender-based violence). ${ }^{6}$ Men and women are also likely to respond to or benefit from social protection mitigation measures in different ways. Thus gender considerations in the social protection response to COVID-19 are important, given both intrinsic

\section{SUMMARY}

As social protection programs and systems adapt to mitigate against the COVID-19 crisis, gender considerations are likely to be overlooked in an urgent effort to save lives and provide critical economic support. Yet past research and learning indicates that small adaptations to make program design and implementation more gender-sensitive may result in overall and equality-related gains. We summarize some of these considerations for LMICs across five areas: 1) Adapting existing schemes and social protection modality choice, 2) targeting, 3) benefit level and frequency, 4) delivery mechanisms and operational features, and 5) complementary programming. It is our hope that responses to COVID-19 will help address, and not exacerbate, pre-existing gender inequalities and lay the groundwork for more gender-sensitive social protection programming in LMICs beyond the crisis, building toward the well-being of societies as a whole.

concerns for the holistic well-being of populations and the fact that longer-term economic development in LMICs is closely linked to women's empowerment.?

Excellent resources with general guidance for responding to COVID-19 through social protection have already been released. ${ }^{8}$ However, more concerted efforts are needed to take gender inequalities into account. Designing gender-sensitive programming can be complex even in settings with strong social protection systems, and doing so is made more challenging by fast-moving timelines and COVID-19 mitigation approaches that complicate delivery and challenge the functioning of social protection systems. However, there are also relatively simple design and implementation adaptations that can make social protection systems more gender-responsive. 


\section{GENDER-SENSITIVITY AND THE EARLY COVID-19 SOCIAL PROTECTION RESPONSE}

While the gender-sensitivity of social protection has been defined in different ways, we broadly consider a continuum whereby programs and systems can be considered more gender sensitive if they "recognize specific needs and priorities of women and men, and seek to purposefully and proactively tackle gender inequalities by questioning and challenging the structures, institutions and norms on which these inequalities are based.. ${ }^{119} \mathrm{Arapid}$ assessment of the gender sensitivity of initial COVID-19 social protection responses (as of April 3, 2020) shows that, out of 418 social protection initiatives, only about 11 percent show some (but limited) gender sensitivity. ${ }^{10}$ For example:

- Six programs specifically target pregnant women or women receiving maternity benefits (Argentina, Armenia, El Salvador, Hungary, Russia, and Sri Lanka).

- Eight programs target women specifically, due to various criteria, including nutritional risk, lack of spouse, women leaders, pre-existing female beneficiaries, or top-ups to programs for women (Argentina, Brazil, Colombia, Egypt, India, Italy, and Pakistan).

- Sixteen programs specifically take into account childcare duties or provide benefits related to childcare (Austria, Cook Islands, Czech Republic, France, Germany, Italy, Norway, Poland, Romania, Serbia, Slovenia, South Korea, Spain, and the United States)

- Two programs are targeted specifically to healthcare workers (who are primarily women), ${ }^{11}$ including covering exposure or injury-related costs and compensation for infection (Philippines) and higher levels of childcare vouchers as compared to the rest of the population (Italy).

- Sixteen programs target informal workers, who are likely to be disproportionately women, through instruments including vouchers for skills training (Indonesia), wage subsidies (Australia), utility subsides (Vietnam), public works for those who lost livelihoods (Philippines), food vouchers (Jordan), and cash transfers (Argentina, Australia, Cabo Verde, Colombia, Ecuador, Morocco, Namibia, North Macedonia, Peru, Philippines, and Tunisia).

\section{LESSONS AND CONSIDERATIONS}

While there is no "one-size-fits-all" approach, based on existing evidence, we summarize key lessons, considerations, and guidance across five areas: 1 ) Adapting existing schemes and social protection modality choice, 2) targeting, 3) benefit level and frequency, 4) delivery mechanisms and operational features, and 5) complementary programming.

\section{Adapting existing schemes and social protection modality choice}

Adapting existing schemes to be contagion-safe is a likely first step for governments, and these adaptations can have gender implications. Modifications to relax requirements on meeting conditionalities (e.g., tied to work, health, or schooling requirements) can simultaneously reduce viral spread and benefit women who may be required to fulfill these conditionalities, be mobility-constrained, or have fewer social and information networks. Additionally, together with bolstering the health infrastructure, it is important to expand access to healthcare via fee waivers or automatic health insurance enrollment to encourage testing and containment of COVID-19.12 These measures may have gendered effects, as maintaining and expanding benefits on an individual level could help counteract gender inequalities in health-seeking behavior. In addition, they may help ensure that women continue to seek care for critical routine maternal and child health services, including access to family planning and reproductive health. ${ }^{13}$
During times of food insecurity, women and children may be the first to reduce food consumption due to intrahousehold inequalities. Thus providing direct cash transfers, in-kind transfers, or food vouchers - all shown to improve food security - can have positive impacts on women and other household members. ${ }^{14}$ Research also shows that economic transfers can improve overall household economic security and emotional well-being, which directly benefit women and can contribute to reducing intimate partner violence. ${ }^{15}$ Modality choice may additionally have gendered impacts. A cross-country qualitative study (Bangladesh, Egypt, El Salvador, Jordan, Mali, and Rwanda) showed that in some settings with restrictive gender norms, foodbased transfers or vouchers help women retain control over resources. ${ }^{16}$ While cash benefits (via e-payments) are still widely recommended due to cost-effectiveness ${ }^{17}$ and their ability to promote dignity and freedom of choice, among other things, implementers should also consider the feasibility of providing in-kind transfers (including bulk, storable, and fortified food or hygiene supplies such as soap). Considering in-kind modalities will be important when mobility is restricted, where markets are limited, where food prices spike, or where supply chains close due to COVID-19 restrictions. ${ }^{18}$

These COVID-19-related market access issues may exacerbate ongoing seasonality and climate issues faced by producers and consumers during lean seasons and periods of drought and other weather shocks. While food and 
hygiene are not inherently gender-sensitive, women are often responsible for daily shopping, cooking, and cleaning. Therefore, providing food and hygiene supplies, if feasible in a safe manner, may decrease women's exposure to unsafe environments or crowds. ${ }^{19}$ Once social-distancing restrictions are relaxed, implementers of public-works programs could allow a grace period for work requirements during recovery. In addition, ensuring that wages are fair, work is dignified, and women are safe and able to participate, including through exemptions for lactating and pregnant women, will be crucial. ${ }^{20}$ When schools reopen, implementers should ensure that economic constraints are eased, including through universal fee waivers and cash for education top-ups, particularly for adolescent girls who may be at heightened risk of dropping out. Research shows that adolescent girls are highly vulnerable to multiple risks during outbreaks. For example, a study in Sierra Leone found that when schools closed due to Ebola, without protective "girl clubs," young girls aged 12 to 17 were twice as likely to be pregnant and less likely to re-enroll when schools opened the following year. ${ }^{21}$

\section{Targeting}

Which households should be targeted for benefits and who in those households should be "named" as recipients are critical questions. Given the practicality of leveraging existing social protection programs, it makes sense to retain the original individual-level targeting of many such benefits (e.g., unemployment insurance). However, many vulnerable people will be excluded by such targeting; for example, unemployment insurance typically does not cover informal workers, including the majority of women, who primarily work in the informal economy. ${ }^{22}$ Providing universal "household-level" transfers in areas severely affected by COVID-19 (i.e., geographical targeting) can help address this issue. However, even household-level transfers have named recipients, and evidence suggests the identity of the named recipient may matter. Moreover, households in LMICs have diverse structures, some of which may raise the need to target multiple beneficiaries within a household - for example, each wife in polygamous households - as evidence from multiple countries in West Africa indicates that effects of cash transfers may differ based on marital structures. ${ }^{23}$

Although evidence is mixed regarding the broader economic and family well-being impacts of the recipient's gender, ${ }^{24}$ a limited number of studies from development settings indicate that naming female recipients may lead to larger improvements in women's empowerment. For example, an experimental study of an unconditional cash transfer in Kenya found larger increases in an index of women's empowerment when transfers were given to women rather than men. ${ }^{25}$ In addition, while no regional statistics exist showing the current sex-disaggregation of social protection benefits, recent reviews of the evidence agree that programs that target women have led to increases in women's well-being across multiple domains. ${ }^{26}$ Although there are fears that targeting cash transfers to women may lead to male backlash and greater risk to women, this is largely not borne out in the development literature. ${ }^{27}$ Fewer rigorous studies exist in humanitarian crisis settings due to contextual constraints; however, some studies indicate complex relationship dynamics. For example, men's feeling unable to fulfill traditional "provider" roles, particularly given limited employment opportunities in these contexts, may contribute to household conflict. ${ }^{28}$

Taken together, we believe the evidence supports considering women as named recipients for benefits if contextually feasible, while recognizing that during particularly acute periods of the crisis (e.g., during lockdowns), household tensions may be extreme. Therefore, in settings where rapid gender analysis has shown feasibility and acceptability of targeting women in the past, we believe there is good evidence suggesting gains from continuing. In contrast, in places where targeting to women was deemed infeasible in the past, we do not recommend challenging these norms explicitly during periods of COVID-19-related crisis.

In the latter scenario, however, minor tweaks to the targeting and messaging around transfers could contribute to greater gender equity, including: (1) authorizing multiple people within a household to carry out transactions or, if this is not possible, asking households to nominate a named recipient rather than automatically assigning the "household head"; (2) providing relevant information to both men and women - for example, on transfer modality or how to access transfers; and (3) providing complementary messaging emphasizing that benefits are for the entire family and meant to encourage joint decision-making and household harmony. ${ }^{29}$

\section{Benefit levels and frequency}

Guidance is clear that benefits in response to COVID-19 should be quick and lumpy to ensure sufficient support before supply chains and systems are overwhelmed, as well as to avoid more frequent payment distributions and contact with others. ${ }^{30}$ However, evidence is mixed on whether benefit levels have gender implications. For example, qualitative studies in Somalia and across other countries (Bangladesh, Egypt, El Salvador, Jordan, Mali, and Rwanda) indicate that smaller transfer values targeted to women may be more likely to remain in their control, but these studies do not directly compare different transfer sizes. ${ }^{31}$ On the other hand, evidence where benefit levels were randomized 
in Kenya and Rwanda suggest that larger cash transfers result in comparatively higher well-being effects for households, and women specifically. ${ }^{32}$ In addition, a study in Nigeria found no overall differences in outcomes for women or households when transfers were given quarterly (lumpy) versus monthly (smoothed), but quarterly transfers were more cost-effective. ${ }^{33}$

Thus the limited evidence suggests a potential tradeoff, in that fewer distributions of larger transfers may lead to lower control and decision-making among women, depending on the context and benefit levels. However, we are not aware of evidence suggesting that larger transfers to women lead to adverse effects. Therefore, we believe that the evidence points toward the primary importance of ensuring that benefit levels are of sufficient value to cover the duration of the COVID-19-related crisis.

Additionally, as care burdens disproportionately fall on women, additional "top ups" to usual benefit levels should be considered for households caring for children who may be out of school and require additional resources (e.g., meals at home, home tutoring, or school supplies). Care for sick family members or relatives due to COVID-19 is also likely to directly fall on women and girls, with additional considerations for time and compensation. Finally, if benefit levels are calculated on a simple per capita basis or on pre-crisis earning levels, it is important to keep in mind that female-headed households are likely to be smaller - and thus may appear better off on a direct per-capita poverty measure - yet may still be more disadvantaged due to economies of scale and reduced access to markets and services, among other reasons. ${ }^{34}$

\section{Delivery mechanisms and operational features}

While delivery mechanisms and operational features may be chosen because they are most logistically feasible in crisis conditions, simple choices may have gender implications. Social protection measures already in place should seek to simplify delivery and operation if possible, which may place fewer burdens on recipients. For example, operational-related conditionalities should be dropped, including those related to health and schooling conditions for which the mother or female guardian may be responsible, as discussed above. In addition, requirements such as re-certifications or graduation assessments could be dropped or postponed. Clear grievance mechanisms should be set up in advance (with remote options available), and implementation and management staffing/coordinating bodies should include women. Programs already using e-payments or direct wire transfers can utilize these features for safe and quick transfer of benefits. ${ }^{35}$ While this may not be an option for many settings, in the longer term, national programs should invest in establishing e-payment mechanisms and encouraging financial inclusion. One study in Niger showed that electronic payments to women (versus manual payments) were spent on better and more diverse food items and led to reduced time spent collecting transfers and travel distances for women. ${ }^{36}$ As mobile transfers were less observable, women were also more able to conceal them from other household members. Extending the network of electronic payments may also lead to financial inclusion if the payments are linked to bank accounts, which will benefit women who have lower rates of financial inclusion. ${ }^{37}$ These benefits must be considered against the trade-off that women are less likely to own or have access to cell phones (mobile money accounts) in many settings; therefore, some implementers have provided cell phones as part of programming that prioritizes mobile transfers.

When transfers are delivered in person, gender-sensitive delivery and labeling may help address concerns around the time burden and limited mobility of women and ultimate control over benefits. For example, simple nudges or behavioral designs at pay points or community meetings are being tested with promising early effects in Kenya, Tanzania, and Madagascar to help women increase goal setting, planning, and self-affirmation. ${ }^{38}$ Information around and delivery of benefits should be practicable from the perspective of both women and men.

If there are concerns that women's access to information is limited, efforts should be made to reach them via platforms that are known to work in the specific context. These could be text messages or community postings, or through radio or television for nonliterate populations. Women's collectives are important existing social networks and sources of information that can be leveraged for generating awareness about the social protection measures in place. ${ }^{39}$ For example, group leaders (who in many contexts are linked to higher-level federations/local governments) could relay information safely through WhatsApp groups, phone messages, and print materials about conditionalities being dropped or about cash or in-kind transfer delivery points and pick-up times. ${ }^{40}$ This is not to propose that women should continue to congregate in groups, but rather that women leaders be tasked with spreading information with support from local governments or NGOs and in ways that are safe. ${ }^{41}$

For direct transfer of benefits, the ideal solution is doorto-door delivery, if feasible. If women (households) must go collect the transfers, then pick-up points should be someplace nearby, with a way to prevent crowding.

Gender-sensitive delivery options should be considered for existing feeding and care programs - such as school meals and early child development centers - if they can be implemented within recommended safety guidelines for COVID-19. In addition, essential workers require 
safe childcare options, and existing child development centers may be well placed to fill this gap. However, government-run schools and early child development centers are often the first to be shut down in response to the pandemic. Local and national NGOs have stepped in to fill some of these gaps. Several local NGOs in India, for example, have started community kitchens to deliver rations and cooked meals to the most vulnerable households and also to quarantined migrants who recently returned from urban centers as a result of the nationwide lockdown. ${ }^{42}$ If operationally successful, these effects can pave the way for utilizing existing platforms for other services, including "school feeding" at home, while serving the dual objective of supporting local women's groups.

\section{Complementary programming}

During the COVID-19 crisis, many complementary activities typically linked to social protection that provide women with information and social support, such as group trainings or home visits on nutrition, may no longer be possible. Instead, other methods of contact and interaction will be needed. There are two main gender considerations when planning complementary programming during COVID-19. First is deciding what type of information, support, and services are most needed and relevant for women (and how critical they are to maintain during the crisis), and second is women's ability to successfully access the information, support, and services.

With respect to type of complementary activities, the most relevant and important for women are likely those focusing on food and nutrition, including ways of accessing or growing nutritious foods when markets and supply chains are down; water and sanitation, as information about hygiene and social distancing is critical for safety and stopping the spread of COVID-19; maternal health, including antenatal care, as travel may be restricted, health services overburdened, and health centers a potential source of contamination; sexual and reproductive health, including family planning and menstrual hygiene management; parenting and learning for children, as many schools are closed, with potential gender inequalities in learning and education investments within families during this time; mental health for both men and women, given that many may experience depression from isolation or loss of employment/income or anxiety over the pandemic and related food shortages; and gender-based violence services, such as access to helplines and referrals, given existing evidence suggesting that pandemics may exacerbate diverse risk factors for violence against women and children. ${ }^{43}$ While feasibility of offering all types of services in many contexts is limited, at a minimum, social protection platforms can explore linkages to existing services or options to integrate light-touch informational components of others.

The main channels for providing information and support services that require the least amount of physical contact and travel during the COVID-19 crisis are via telephone, internet, television, and radio. While television and the internet are widespread and have higher functionality in high-income countries, phone and radio are likely to be the best options in LMICs. For example, one-on-one support services for maternal health or mental health could be delivered as "televisits" through online platforms, phone calls from experts, hotlines for women or men to call in and speak with an expert, or live-messaging (such as WhatsApp). ${ }^{44}$

For more generalized messages, television, radio, SMS or voice messages are another way to reach people at scale; however, studies of digital technology across different interventions and settings have produced mixed results. ${ }^{45}$ For example, while mobile phones are ubiquitous and a promising way to continue to provide information and support services, a recent evaluation of a mobile health and nutrition program in Ghana and Tanzania (mNutrition) identifies several challenges to the mobile-phone messaging approach that should be considered. ${ }^{46}$ First, while most households have a cell phone, women have less access to it than men. Second, women may not be able to pay for the service, so waiving any associated fees will be critical. Third, women may be illiterate and not able to read text messages; thus voice messages may be preferable to text messages and phone calls may be preferable to live-messaging. Lastly, where possible, it is important to consider the best time of day for women to receive messages via phone (or radio), given the multiple demands on women's time and that they may not always be carrying a mobile phone (or near a radio). Thus, while mobile approaches may be ideal during the COVID-19 crisis, they must be carefully implemented and combined with broader community-level approaches that include radio and television, which may be better suited to reaching a greater number of individuals.

\section{CONCLUDING THOUGHTS}

As social protection programs and systems adapt to mitigate against the COVID-19 crisis, gender considerations are likely to be overlooked in an urgent effort to save lives and provide critical economic support. Yet past research and learning indicates that small adaptations to make program design and implementation more gender-sensitive may result in overall and equality-related gains. We summarize some of these considerations for LMICs across five areas: 1) adapting existing schemes and social protection modality choice, 2) targeting, 3) benefit level and frequency, 4) 
delivery mechanisms and operational features, and 5) complementary programming.

Decisions around gender-sensitive design and implementation should be, where possible, informed by rapid assessments from the outset, ideally building on gender analysis and sensitivity of existing schemes. ${ }^{47}$ While we are in unprecedented times, policymakers should consider that the gender implications of COVID-19 are expected to be realized over the long term - and thus using social protection to explicitly tackle vulnerabilities beyond the short-term is encouraged. For example, longer-term economic impacts could lead to further informalization of women's work or loss of additional fiscal resources for social protection and essential public services. This is particularly important for groups at risk of life-cycle vulnerabilities, including adolescent girls and women who lose partners to COVID-19. In addition, gender is only one of the many dimensions of inclusion that need attention. Other important aspects include age (partly highlighted above in the life-cycle vulnerabilities), disabilities, and race and ethnicity - all of which should be considered when responding to a pandemic. ${ }^{48}$

Additionally, important gender-related considerations may go beyond the scope of our discussion here - including issues of political economy, coordination, and financing - and should be explored in further guidance. ${ }^{49}$ Because these are complex issues and unintended consequences of programming are possible, more research is needed on intersections of social protection, gender, and pandemics, where ethically feasible. At a minimum, monitoring statistics should be sex- and age-disaggregated and, where possible, measures should be collected to ensure risks to beneficiaries do not increase. ${ }^{50}$ Taken together, these adjusted policy responses and new evidence can lay the groundwork for more gender-sensitive social protection systems in LMICs, both during the crisis and beyond.

\section{FURTHER GUIDANCE:} Gender-sensitive, shock-responsive social protection and emergency cash transfers

Food and Agriculture Organization (2018). Three-part toolkit, consisting of technical guides for gender-sensitive social protection in LMICs: (1) Introduction to gender-sensitive social protection programming to combat rural poverty: Why is it important and what does it mean?, (2) Integrating gender into the design of cash transfers and public works programmes, (3) Integrating gender into implementation and monitoring and evaluation of cash transfers and public works programmes.

Overseas Development Institute (2018-19). Series on "Promoting gender equality and women's empowerment in shock-responsive social protection," including: Working Paper, webinar, and summary blog (part 1 and part 2).

Cash Learning Partnership (CaLP) (2020). Gender and inclusion page focused on humanitarian cash transfers, including tools on safeguarding and protection concerns in operations and delivery: (1) Compendium on Cash \& Voucher Assistance and Gender-Based Violence (CARE), (2) Toolkit on Mainstreaming Gender-Based Violence Considerations in Cash and Voucher Assistance, and CVA in GBV Prevention and Response (WRC, Mercy Corps, and IRC).

Melissa Hidrobo and Neha Kumar are Senior Research Fellows, and Shalini Roy is a Research Fellow, in the Poverty, Health, and Nutrition Division of the International Food Policy Research Institute (IFPRI). Tia Palermo is an Associate Professor in the Department of Epidemiology and Environmental Health, University of Buffalo (State University of New York; SUNY). Amber Peterman is an Associate Research Professor in the Department of Public Policy at the University of North Carolina at Chapel Hill. This work was undertaken as part of the CGIAR Research Program on Policies, Institutions, and Markets (PIM), led by IFPRI. Authors are listed in alphabetical order to denote equal contribution.

We thank Dan Gilligan, Rebecca Holmes, Marie Ruel, John McDermott, and an anonymous reviewer for helpful comments on a previous version.

This publication has been peer reviewed. Any opinions stated in this brief are those of the author(s) and are not necessarily representative of or endorsed by IFPRI, PIM, the University of Buffalo (SUNY), or the University of North Carolina at Chapel Hill. 


\section{Endnotes}

1 U. Gentilini et al., "Social Protection and Jobs Responses to COVID19: A Real-Time Review of Country Measures" ("Living Paper" version 3, April 3, 2020).

2 According to the International Labour Organization, social protection is defined as "the set of policies and programs designed to reduce and prevent poverty and vulnerability throughout the life cycle." This includes contributory schemes (e.g., insurance, labor market policies) as well as noncontributory schemes (e.g., social assistance, social welfare services). While we take a broad view of social protection here, we recognize that most LMICs have low coverage of existing schemes, and in times of crisis will rely primarily on noncontributory social assistance (e.g., cash, in-kind transfers, fee waivers and subsidies).

3 N. Jones and R. Holmes, "Why Is Social Protection Gender-blind? The Politics of Gender and Social Protection," IDS Bulletin 42, no. 6 (2011): 45-52; World Bank, Social Safety Nets and Gender: Learning from Impact Evaluations and World Bank Projects (Washington, DC, 2014).

4 M. Molyneux, "Mothers at the Service of the New Poverty Agenda: Progresa/Oportunidades, Mexico's Conditional Transfer Programme," Social Policy Administration 40, no.4 (2006): 425-49.

5 C. Wenham et al., "COVID-19: The Gendered Impacts of the Outbreak," Lancet 395, no. 10227 (2020): 846-48.

6 UN (United Nations), "The Impact of COVID-19 on Women," Policy Brief (9 April 2020); A. Peterman et al., "Pandemics and Violence Against Women and Children," Center for Global Development Working Paper 528 (April 1, 2020); T. Riley et al., "Estimates of the Potential Impact of the COVID-19 Pandemic on Sexual and Reproductive Health in Low- and Middle-Income Countries," Comment, International Perspectives on Sexual and Reproductive Health 46 (April 2020): 73-76; I. Risso-Gill and L. Finnegan, "Children's Ebola Recovery Assessment: Sierra Leone," Save the Children, World Vision, Plan International, and UNICEF, n.d.

7 World Bank, World Development Report 2012: Gender Equality and Development (Washington, DC: World Bank Group, 2011).

8 See Socialprotection.org's online knowledge hub, among others.

9 FAO (Food and Agriculture Organization of the United Nations), "FAO Technical Guide 1 - Introduction to Gender-Sensitive Social Protection Programming to Combat Rural Poverty: Why Is It Important and What Does It Mean?" (Rome, 2018).

10 Gentilini et al., "Social Protection and Jobs Responses." Note that the number of countries does not always equate to the numerical tally, as some countries have multiple programs counted. While more comprehensive assessments must be done to investigate details of each policy - and we recognize that system-level considerations would assess how programs work together to address gender (rather than individually) - given the low percentage of programs that appear to consider gender at face value, our conservative conclusion is that most programs are unlikely to be gender-sensitive.

11 M. Boniol et al., "Gender Equity in the Health Workforce: Analysis of 104 Countries," Health Workforce Working Paper 1, WHO (World Health Organization), March 2019.

12 WHO, OECD (Organization for Economic Co-operation and Development), and the World Bank, Delivering Quality Health Services: A Global Imperative for Universal Health Coverage (Geneva, 2018).
13 C. Menendez et al., "Ebola Crisis: The Unequal Impact on Women and Children's Health," Lancet Global Health 3, no. 3 (2015): PE130; Riley et al., "Estimates of the Potential Impact of the COVID-19 Pandemic."

14 A.P. de la O Campos and E. Garner, "Women's Resilience to Food Price Volatility: A Policy Response" (FAO: Rome, 2020); M. Hidrobo et al., "Cash, Food, or Vouchers? Evidence from a Randomized Experiment in Northern Ecuador," Journal of Development Economics 107 (2014): 144-56; C. Brown et al., "Most of Africa's Nutritionally Deprived Women and Children Are Not Found in Poor Households," Review of Economics and Statistics 101, no. 4 (2019): 631-44; A. Peterman et al., "Toward Gender Equality: A Critical Assessment of Evidence on Social Safety Nets in Africa," RESAKSS Annual Trends and Outlook Report (Washington, DC: IFPRI, 2019).

15 A.M. Buller et al., "A Mixed-Method Review of Cash Transfers and Intimate Partner Violence in Low- and Middle-Income Countries," World Bank Research Observer 33, no. 2 (August 2018): 218-58.

16 WFP (World Food Programme), The Potential of Cash-Based Interventions to Promote Gender Equality and Women's Empowerment: A Multi-Country Study (February 2019).

17 A. Margolies and J. Hoddinott, "Costing Alternative Transfer Modalities," Journal of Development Effectiveness 7, no. 1 (2015): 1-16.

18 WFP, "Comparative Review of Market Assessments, Methods, Tools, Approaches and Findings," 2013; WFP, "COVID-19: Potential Impact on the World's Poorest People: A WFP Analysis of the Economic and Food Security Implications of the Pandemic," April 8, 2020.

19 A. Golay and S. Tholstrup, "CVA in COVID-19 Contest: Guidance from the CaLP Network," Cash Learning Partnership, April 23, 2020.

20 FAO, FAO Technical Guide 2: Integrating Gender into the Design of Cash Transfer and Public Works Programmes (Rome, 2018).

21 O. Bandiera et al., "Empowering Adolescent Girls in a Crisis Context: Lessons from Sierra Leone in the Time of Ebola," Policy Brief Issue 34 (Gender Innovation Lab and International Growth Centre), World Bank, 2019.

22 UN Women, "Women in Informal Economy," webpage.

23 R. Heath, "Cash Transfers, Polygamy, and Intimate Partner Violence: Experimental Evidence from Mali," Journal of Development Economics 143 (2020): 102410; Peterman et al., "Toward Gender Equality."

24 J. Yoong, L. Rabinovich, S. Diepeveen, "The Impact of Economic Resource Transfers to Women versus Men: A Systematic Review," Technical Report, EPPI-Centre, Social Science Research Unit, University of London, January 2012.

25 J. Haushofer and J. Shapiro, "The Short-Term Impact of Unconditional Cash Transfers to the Poor: Experimental Evidence from Kenya," Quarterly Journal of Economics 131, no. 4 (2016): 1973-2042.

26 Peterman et al., "Toward Gender Equality."

27 A.M. Buller et al., "A Mixed-Method Review of Cash Transfers and Intimate Partner Violence in Low- and Middle-Income Countries," World Bank Research Observer 33, no. 2 (2018): 218-58.

28 WFP, "Potential of Cash-Based Interventions."

29 WFP, "Potential of Cash-Based Interventions." 
30 IPC-IG (International Policy Centre for Inclusive Growth), "How Can Social Protection Systems Respond to the COVID-19 Crisis?" (2020).

31 K. Wasilkowska, "Gender Impact Analysis: Unconditional Cash Transfers in South Central Somalia," Somalia Cash Consortium (December 2012); WFP, "Potential of Cash-Based Interventions."

32 Haushofer and Shapiro, "Short-Term Impact of Unconditional Cash Transfers to the Poor"; C. Mclntosh and A. Zeitlin, "Benchmarking a Child Nutrition Program against Cash: Experimental Evidence from Rwanda" (working paper, June 15, 2018).

33 G.G. Bastian, M. Goldstein, and S. Papineni, "Are Cash Transfers Better Chunky or Smooth?: Evidence from an Impact Evaluation of a Cash Transfer Program in Northern Nigeria," Gender Innovation Lab Policy Brief no. 21, Washington, DC, World Bank Group, n.d.

34 C. Brown and D. van de Walle, "Headship and Poverty in Africa," Center for Global Development Working Paper no. 531, April 14, 2020.

35 A. Gelb and A. Mukherjee, "COVID-19: How Countries Can Use Digital Payments for Better, Quicker Cash Transfers," Center for Global Development blog post, April 6, 2020.

36 J.C. Aker et al., "Payment Mechanisms and Antipoverty Programs: Evidence from a Mobile Money Cash Transfer Experiment in Niger," Economic Development and Cultural Change 65, no. 1 (2016): 1-37.

37 C. Robino et al., "Financial Inclusion for Women: A Way Forward," G20 Insights, policy brief, August 7, 2018; updated October 24, 2019.

38 World Bank and Ideas42, "Behavioral Nudges for Cash Transfer Programs in Madagascar," n.d.; Ideas42, "Cash and Change: Using Behavioral Insights to Improve Financial Health in Three Cash Transfer Programs," September 2019.

39 Brody et al., "Economic Self-Help Group Programs for Improving Women's Empowerment: A Systematic Review," Campbell Systematic Reviews 19 (2015); N. Kumar et al., "Social Networks, Mobility, and Political Participation: The Potential for Women's Self-Help Groups to Improve Access and Use of Public Entitlement Schemes in India," World Development 114 (February 2019): 28-41.

40 C.S. Reddy, "SHG Federation: An Institutional Innovation to Sustain SHGs," NewsReach, September-October 2012.

41 World Bank, "In India, Women's Self-Help Groups Combat the COVID-19 (Coronavirus) Pandemic," feature story, April 11, 2020.

42 World Bank, "In India, Women's Self-Help Groups Combat the COVID-19 (Coronavirus) Pandemic."

43 WFP, "General Guidelines for Food and Nutrition Assistance in COVID-19 Outbreak," General Guidelines, version 1, March 6,
2020; L. Duan and G. Zhu, "Psychological Interventions for People Affected by the COVID-19 Epidemic," Lancet Psychiatry 7, no. 4 (2020): 300-302; UNICEF, "Monitoring and Mitigating the Secondary Impacts over the Coronavirus Disease (COVID-19) Pandemic on WASH Services Availability and Access," UNICEF technical note, March 11, 2020; UNFPA (United Nations Population Fund), "Sexual and Reproductive Health and Rights: Modern Contraceptives and Other Medical Supply Needs, Including for COVID-19 Prevention, Protection and Response," Coronavirus Disease (COVID-19) Preparedness and Response UNFPA Interim Technical Brief, March 23, 2020; UNFPA, "COVID-19 Technical Brieffor Maternity Services," April 2020; S. Garçon, "COVID-19 Has Made Menstrual Health More Urgent Than Ever," PSI (Population Services International), March 8, 2020; Peterman et al. "Pandemics and Violence Against Women and Children."

44 P. Webster, "Virtual Health Care in the Era of COVID-19," Lancet 395, no. 10231 (2020): 1180-1181.

45 V. Stephani, D. Opoku, and W. Quentin, "A Systematic Review of Randomized Controlled Trials of mHealth Interventions against Non-Communicable Diseases in Developing Countries," BMC Public Health 16, no. 572 (2016); I. Barnett et al., "Dial " N" for Nutrition? A Landscape Analysis of What We Know about m-Nutrition, m-Agriculture and m-Development," IDS (Institute of Development Studies) Working Paper, no. 481 (2016); S.H. Lee et al., "Effectiveness of mHealth Interventions for Maternal, Newborn and Child Health in Low- and Middle-Income Countries: Systematic Review and MetaAnalysis," Journal of Global Health 6, no. 1 (2016): 1-17.

46 I. Barnett and B. Faith, "Evaluating the mNutrition Programme: What Have We Learnt So Far?," IDS Opinion, April 25, 2019.

47 FAO, "FAO Technical Guide 1."

48 "Disability Inclusive Social Protection Response to the COVID-19 Crisis," brief prepared in the frame of the UNPRPD funded project on inclusive social protection for the empowerment of persons with disabilities implemented by ILO and UNICEF in close collaboration with the International Disability Alliance.

49 R. Holmes et al., "The Politics of Gender-Responsive Social Protection," Working Paper 568, Overseas Development Institute, November 2019; A.C. Romano, "Financing Gender-Responsive Social Protection," Socialprotection.org blog, Social Protection and Gender Equality Series, October 10, 2019.

50 S. Bloom et al., "Guidance for Gender Based Violence (GBV) Monitoring and Mitigation within Non-GBV Focused Sectoral Programming," CARE, October 2014.

\section{INTERNATIONAL FOOD POLICY RESEARCH INSTITUTE A world free of hunger and malnutrition IFPRI is a CGIAR Research Center}

1201 Eye St, NW, Washington, DC 20005 USA | T. +1-202-862-5600 | F. +1-202-862-5606 | Email: ifpri@cgiar.org | www.ifpri.org | www.ifpri.info DOI: https://doi.org/10.2499/9780896293793 